\title{
Recurrent opticomyelitis associated with anti-DNA antibody
}

\author{
J Kira, I Goto
}

\begin{abstract}
Two patients had frequent attacks of acute myelitis and optic neuritis. One patient lacked any other organ involvement whereas the other developed systemic manifestations of systemic lupus erythematosus 14 years after the onset. Both patients developed antinuclear and antidouble strand DNA antibodies after the onset of neurological involvement. These patients, whose neurological manifestations were indistinguishable from multiple sclerosis, were thus considered to have systemic lupus erythematosus related collagen disease.
\end{abstract}

(F Neurol Neurosurg Psychiatry 1994;57:1124-1125)

Neurological manifestations often appear in patients with systemic lupus erythematosus. ${ }^{12}$ In most cases, however, neurological involvement occurs during the active phase associated with a widespread dissemination of the disease. ${ }^{12}$ On the other hand, Fulford $e t \mathrm{al}^{3}$ described a distinct variant of systemic lupus erythematosus as lupoid sclerosis. In this condition, chronic progressive spastic paraplegia was a main feature with minimal evidence of any involvement with other organs. We describe two patients with such a condition but who both had an unusual course.

\section{Case reports}

CASE 1

The patient, a 43 year old woman, noted numbness in her left foot and difficulty walking at 27 years of age. At that time, she was found to be normal in a general physical examination, but a neurological examination showed moderate hyper-reflexia and hypaesthesia in the lower limbs with Babinski sign on the right side. Dexamethasone alleviated her symptoms. Over the next 14 years, she experienced three episodes of optic neuritis, two episodes of acute transverse myelitis affecting either the C5 or T8 levels, and left hemiparesis once, with a favourable outcome under prednisolone treatment. Most episodes were accompanied by mild pleocytosis of the CSF ( 7 to 37 mononuclear cells $/ \mathrm{mm}^{3}$ ). Tests for lupus erythematosus and antinuclear antibody (ANA) in the patient's serum were negative at 29 years of age; ANA became positive at 1:64 for the first time at 32 years of age, whereas the lupus erythematosus test remained negative.

At 41 years of age, a rapid loss of visual acuity developed in both eyes. A physical examination showed a malar rash and a decrease in the arterial pulse in her right popliteal and pedal arteries, as well as bilateral blindness with optic atrophy, mild weakness of the left upper limb, and generalised hyper-reflexia with Babinski sign bilaterally. Increased ESR $(68 \mathrm{~mm} / \mathrm{h})$ and lymphopenia $(540 / \mu 1)$ were also seen. The lupus erythematosus test was positive and ANA was present at 1:640 (speckled type). Antidouble strand (ds) DNA antibody was negative. Examination of CSF showed mild pleocytosis and positive oligoclonal bands. Intravenous digital subtraction angiography showed an occlusion of the right internal carotid and right common iliac arteries. With pulse treatment of methylprednisolone ( $1 \mathrm{~g} /$ day for three days), then oral prednisolone, the laboratory findings returned to normal, but the visual acuity only minimally improved.

At 42 years of age, the patient experienced a recurrence of acute transverse myelitis affecting the T5 level; ANA (1:160), anti-ds DNA antibody $(4 \cdot 1 \mathrm{U} / \mathrm{ml}$; normal $<2)$, and lupus erythematosus test were positive. The serum C4 decreased to $8 \mathrm{mg} / \mathrm{dl}$ (normal 11-46), but the $\mathrm{C} 3$ and $\mathrm{CH} 50$ were normal. MRI of the spinal cord showed atrophy of the midthoracic cord. Acute traverse myelitis was resolved by methylprednisolone leaving only a mild sensory impairment in the lower limbs.

CASE 2

The patient, a 41 year old woman, noted paraesthesia in the left hand and difficulty in walking and micturition at 22 years of age. Examination of CSF showed 48 mononuclear cells $/ \mathrm{mm}^{3}$ and a protein concentration of 96 $\mathrm{mg} / \mathrm{dl}$. Oral betamethasone rapidly alleviated her symptoms. Over the next 15 years she had 10 more episodes of similar acute myelitis affecting the cervical cord and once had a bout of right optic neuritis. When she was first referred to our clinic at 35 years of age, an ANA and lupus erythematosus test were both negative.

At 38 years of age, she again developed acute myelitis at the cervical level. Her ANA 
titre became positive at 1:80 (speckled type), but the lupus erythematosus test and anti-ds DNA antibody were negative. Her CSF showed 7 mononuclear cells $/ \mathrm{mm}^{3}$, a protein concentration of $107 \mathrm{mg} / \mathrm{dl}$, and $\mathrm{IgG}$ index 0.74 (normal <0.65). MRI showed hyperintensity lesions in the swollen cervical cord (C2 to C7), and hypothalamus and anterior commissure on both sides on the T2 weighted images with enhancement by gadolinium-DTPA. Prednisolone alleviated her manifestations and the ANA became negative.

At 40 years of age, right optic neuritis recurred, ANA was present at 1:160 (speckled type), and an anti-ds DNA antibody test was also positive $(3.5 \mathrm{U} / \mathrm{ml})$. A lupus erythematosus test was still negative and C3, C4, and $\mathrm{CH} 50$ were normal. Methylprednisolone pulse treatment gradually alleviated the right visual impairment leaving a large central scotoma.

In both patients, serological tests for syphilis, rheumatoid factor, anti-ribonucleoprotein, anti-Sm, anti-Ki, antimitochondria, anti-SSA, anti-SSB, and anticardiolipin antibodies were negative, whereas the partial thromboplastin time and the activated partial thromboplastin time were normal. The HLA types were A2, A24, B46, B54, Cw1, and DR8 in case 1 and A24, B52, B61, Cw3, DR2, and DR12 in case 2.

\section{Discussion}

Both of the patients were initially diagnosed as having relapsing-remitting multiple sclerosis, as they lacked any clinical or laboratory findings of systemic lupus erythematosus. Long after the onset of neurological manifestation, case 1, however, finally met the current diagnostic criteria for systemic lupus erythematosus. ${ }^{4}$ Similarly, the presence of both ANA and anti-ds DNA antibody was strongly suggestive of systemic lupus erythematosus related collagen disease in case 2 .

Interestingly, our patients were remarkably unlike the usual form of systemic lupus erythematosus in several important aspects. Firstly, these patients lacked any clinical or laboratory findings characteristic of systemic lupus erythematosus until either 14 or 18 years after the onset of neurological involvement. Secondly, they had frequent attacks of myelitis and optic neuritis. In systemic lupus erythematosus, the coexistence of myelitis and optic neuritis in a single patient is exceedingly rare, ${ }^{5-7}$ and, the frequent recurrence of such involvement has never previously been reported.

Our patients had a disease resembling lupoid sclerosis in that the neurological manifestation was a main feature with either no or only minimal evidence of other organ involvement. ${ }^{3}$ Whereas the cases of Fulford et al commonly showed chronic progressive spastic paraparesis, ${ }^{3}$ our patients instead showed a relapsing-remitting course of the neurological deficits and an involvement of both the optic nerves and spinal cord. Moreover, in the patients of Fulford et al, false positive syphilis serology was prominent, whereas our patients showed neither a false positive syphilis serology nor anticardiolipin antibodies.

We have found no other patients showing any close similarities to our cases in published reports, although there have been two case reports of recurrent myelitis occurring in patients with no other clinical features of systemic lupus erythematosus, but who did present with anti-DNA antibodies instead. ${ }^{89}$ Taking into account the similarities between these two cases and our patients, there may be a subgroup of patients showing recurrent myelitis and optic neuritis as a main feature associated with anti-DNA antibodies, although it is still possible that multiple sclerosis and systemic lupus erythematosus may, in fact, coexist. Therefore, we propose that the long term finding of an autoantibody profile in patients with relapsing-remitting multiple sclerosis should be considered.

1 Khamashta MA, Cervera R, Hughes GRV. The central nervous system in systemic lupus erythematosus. nervous system in systemic

2 Futrell N, Schultz LR, Millikan C. Central nervous system disease in patients with systemic lupus erythematosus. Neurology 1992;42:1649-57.

3 Fulford KWM, Catterall RD, Delhanty J, Doniach D, Kremer M. A collagen disorder of the nervous system presenting as multiple sclerosis. Brain 1972;95:373-86.

4 Tan EM, Cohen AS, Fries JF, et al. The 1982 revised criteria for the classification of systemic lupus erythematosus. Arthritis Rheum 1982;25:1271-7.

5 April RS, Vansonnenberg E. A case of neuromyelitis optica (Devic's syndrome) in systemic lupus erytheoptica (Devic's syndrome) in systemic lupus erytheliterature. Neurology 1976;26:1066-70.

6 Kinney EL, Berfoff RL, Rao NS, Fox LM. Devic's syndrome and systemic lupus erythematosus: a case report drome and systemic lupus erythematosus:
with necropsy. Arch Neurol 1979;36:643-4.

7 Kenik JG, Krohn K, Kelly RB, Bierman M, Hammeke MD, Hurley JA. Transverse myelitis and optic neuritis in systemic lupus erythematosus: a case report with magnetic resonance imaging findings. Arthritis Rheum 1987;30:947-50

8 Yamamoto $M$. Recurrent transverse myelitis associated with collagen disease. $\mathcal{F}$ Neurol 1986;233:185-7.

9 Lindsey JW, Albers GW, Steinman L. Recurrent transverse myelitis, myasthenia gravis, and autoantibodies. Ann Neurol 1992;32:407-9. 